\title{
Apuntes sobre evaluación en talleres de artes visuales del nivel superior.
}

\section{Notes on evaluation in visual arts workshops of the superior level.}

\begin{abstract}
Víctor Alejandro Guillén García
Estudiante de Doctorado, Facultad de Filosofía y Letras, Universidad Autónoma de Nuevo León. (México).
\end{abstract}

CE: alejandroguillengarcia42@gmail.com / ID ORCID: 0000-0002-7603-3648

\section{Irma María Flores Alanís, Profesora e Investigadora}

Facultad de Filosofía y Letras, Universidad Autónoma de Nuevo León. (México).

CE: irma.floresal@uanl.edu.mx / ID ORCID: 0000-0002-4914-3091

\section{Esta obra está bajo una Licencia Creative Commons Atribución-NoComercial 4.0 Internacional}

Recibido: $11 / 09 / 2020$

Revisado: $18 / 03 / 2021$

Aprobado: 22/30/2021

\section{RESUMEN}

La educación para la formación de artistas, fuera del formato de academias del siglo XIX, es una actividad que a partir de la segunda mitad del siglo XX ha sido insertada en los currículos y programas de estudio formales, desde entonces, se han sumado cuestionamientos e inquietudes sobre cuáles son las mejores estrategias de enseñanza y de evaluación sobre los procesos de investigación/creación abordados en talleres de artes (debido a que, difícilmente, la creación artística, se consideraa investigación), lo anterior 
presenta un reto: objetivar con sentido crítico, no solo productos y resultados, sino también las prácticas y los significados a cuestiones que, por su constante evolución y naturaleza, pueden tornarse subjetivas.

El artículo presente, nace de las observaciones realizadas en durante el semestre enero junio de 2019 en un programa de artes visuales del nivel superior en México, se fundamenta en la exploración y análisis de estudios previos sobre la educación y evaluación en disciplinas artísticas, para analizar información que aporte ideas que clarifiquen aspectos susceptibles a evaluarse observando procesos de Investigación y Creación artística (IC) nacidos en programas escolarizados; las posibilidades y responsabilidades implícitas, no sólo a los estudiantes, sino también a profesores y cuerpos directivos, incidiendo a través de su práctica, en la sociedad que los abraza y sustenta.

Palabras clave: Evaluación formativa. Educación artística. Artes visuales. Investigación. Creación artística.

\section{ABSTRACT}

Education for the training of artists, outside the format of academies of the 19th century, is an activity that from the second half of the 20th century has been inserted in the curricula and formal study programs, since then, questions and Concerns about which are the best teaching and evaluation strategies regarding the research / creation processes approached in arts workshops (because artistic creation is hardly considered research), the above presents a challenge: to objectify with a critical sense, not only products and results, but also the practices and meanings of issues that, due to their constant evolution and nature, can become subjective.

This article is born from the observations made during the January-June 2019 semester in a visual arts program of the higher level in Mexico, is based on the exploration and analysis of previous studies on education and evaluation in artistic disciplines, to analyze information that provides ideas that clarify aspects that can be evaluated by observing processes of Research and Artistic Creation (Cl) born in school programs; the implicit possibilities and responsibilities, not only to students, but also to teachers and governing bodies, influencing through their practice, the society that embraces and supports them.

Keywords: Formative assessment. Arts education. Visual arts. Research. Artistic creation. 


\section{Introducción}

El aprendizaje y la práctica artística fuera de Academias, son procesos relativamente recientes, producto de la ruptura que se manifiesta a principios del siglo XIX en la cultura de occidente, donde la enseñanza de las conocidas como "artes mayores" (Pintura, Escultura, Literatura y Arquitectura) comienza a salir de la estructura tradicional heredada del Renacimiento (Storr, 2009), donde las actividades se desarrollaban al interior de un estudio-taller, por lo general del artista experto, estructuradas en torno a la instrucción, comprensión y dominio de técnicas y habilidades, con base a modelos clásicos (Grecia y Roma), transmitidas de maestro a sus aprendices.

A partir de dicha ruptura, con el apogeo de las universidades durante el siglo XX, se inicia un proceso para integrar, técnicas y habilidades prácticas, con dominios cognitivos fundamentados en la teoría, con el fin de asimilar y producir nuevos conocimientos, dentro de la normatividad de los planes de estudios.

En la actualidad la evaluación formativa en talleres de producción en artes visuales enfrenta retos nacidos en la naturaleza misma de la disciplina, ya que al ser un proceso que pretende conocer tanto habilidades, destrezas y dominio de materiales; como la evidencia de conocimientos, reflexión, innovación y creatividad, la balanza suele inclinarse al área de la percepción visual, superando el valor de la carga conceptual, especialmente en un mundo con predilección de "imágenes poderosas" (Bauman, 2005): anuncios publicitarios, objetos reproducibles en escala masiva, tendencias de la moda, y otros soportes preparadas para el consumo inmediato.

Michaud (2007) escribe con este respecto la existencia de dos grandes grupos donde se mueve la manifestación artística en el siglo XXI; uno donde se rinde culto, casi de adoración a la belleza prefabricada, el triunfo de la estética profusa, a la experiencia estética pasajera y sin soportes, casi gaseosa; y en otro extremo, pero con el mismo resultado, la saturación y reproductibilidad seriada del objeto artístico, la transformación de los museos y galerías en supermercados del arte. Con el seguimiento de dichas líneas, la evaluación del objeto artístico, puede sesgarse hacia la estandarización de tendencias esteticistas para consumo (real o abstracto) de los espectadores (Spentsas, 2017, p.218). 
La evaluación realizada de tal forma, o en el caso presente, del objeto artístico, suele verse influenciada por los gustos o experiencias previas del profesor-espectador (Romero, 2011), o a la estandarización de tendencias esteticistas para consumo masivo. En el caso específico del sistema educativo, el nivel superior, dicha responsabilidad recae en primera línea en el profesor de asignatura, con el compromiso que se le adjudica desde los parámetros establecidos por el programa educativo, planes de estudio y políticas institucionales, en conjunción con el criterio, experiencia y gustos personales del profesor responsable de la evaluación.

El presente artículo, nace del interés desde la práctica profesional y de las observaciones realizadas en campo, en una búsqueda por conocer cuáles son los indicadores que se consideran relevantes al evaluar la producción artística en talleres, de los estudiantes en el nivel superior, los retos a los que se enfrentan los profesores y las posibles alternativas para reducir la brecha entre teoría, práctica y apreciación de las expresiones artísticas. No se ha de olvidar que los juicios estéticos corresponden a contextos y realidades matizadas por la cultura y el conocimiento del grupo social donde la misma se realiza, por tanto, la complejidad puede tornarse mayor.

\section{Desarrollo}

Para entender cuando se habla de talleres teórico-prácticos en el campo de estudio (nivel superior), se hace referencia al trabajo conjunto y colaborativo entre grupos de alumnos, con supervisión de un profesor, para llevar a cabo actividades que promuevan el aprendizaje mediante la ejecución "El aula taller constituye un escenario para aprender haciendo" (De Vincenzi, 2009, p.42). Ese "aprender haciendo" tiene además la característica que se lleva a cabo de una manera colaborativa, donde estudiantes intercambian ideas, propuestas, métodos e inquietudes; el maestro en tal contexto es el encargado de proponer un problema o situación a abordar, apoyando con propuestas de aspectos teóricos y metodológicos, así como de bibliografía que de aportes para la construcción de las habilidades y/o saberes buscados.

De manera complementaria desde el pensamiento de Liv Mjelde, es posible considerar que es en lo talleres donde "el aprendizaje acontece en una comunidad de práctica social en la cual 
predomina la cooperación y el sentimiento de que lo que está siendo producido es una obra colectiva." (Mjelde, 2015). Es en los talleres (aula-taller) donde se propicia la dinámica que favorece a las construcciones procedimentales a través de la acción, el uso del cuerpo en conexión con la mente, es la oportunidad entonces de involucrar todos los sentidos, para la construcción de ideas y aprendizajes.

Para analizar cuáles son las mejores estrategias de enseñanza y de evaluación sobre los procesos de investigación/creación abordados en talleres de artes, se partió de los principios de la Teoría Fundamentada (en adelante TF); que buscan esa visión epistémica, multidimensional del proceso creativo y de su evaluación, y que emerge de los "datos recopilados de manera sistemática y analizados por medio de un proceso de investigación" (Strauss y Corbin, 2002).

El primer paso, fue trabajar con la observación participante en los talleres del Departamento de Artes Visuales de una universidad del centro del país en México, donde la recogida de datos se realizó a través de un diario de campo, bitácora y de entrevistas a expertos, revelando las primeras variables del estudio, llevando al investigador a originar proposiciones teóricas, basadas en la observación para la obtención de datos empíricos (Jiménez-Fontana, García-González, Azcárate, Navarrete y Cardeñoso, 2016), obtenidos directamente en el ambiente natural.

La observación participante, se desarrolló en los momentos del curso/taller, para entender y analizar las diferentes interacciones, dinámicas, estrategias didácticas y herramientas para la evaluación, y recabar datos que demuestren los criterios sobre si se da una evaluación formativa y qué es lo que toma en cuenta el profesor: los conocimientos, las habilidades, la creatividad, entre otros, que se ven implicados en los procesos de IC.

A través de la observación participante se logró recuperar: el tipo de evaluación, si corresponde a los procesos (formativa), a los productos (sumaria), y si se presenta algún sesgo del profesor, derivados de interferencias ajenas al proceso educativo. Para el análisis de resultados derivados de la observación, se utilizó el programa de Atlas ti 8.4.4, que arrojó las Unidades de Observación (UO) de donde se derivó la formación de tres grupos: Profesores, Estudiantes y Proceso Creativo. 


\section{Proceso Creativo}

Graham Wallas (1926) es a quien se le atribuye el primer manual sobre los elementos y pasos que sigue un proceso creativo: preparación, incubación, iluminación y verificación, si bien, es el primer referente, y ha servido como fundamento para entender la evolución del proceso creativo; el modelo de Wallas es lineal, y se traduce el entendimiento parcial de un sistema que se ha complejizado, como resultado de los cambios en la manera de enseñar, de aprender y de evaluar, en el siglo XXI.

Para esclarecer el concepto Herrán (2014) menciona que para valorar la creatividad es necesario, separarle de sus resultados, porque estos pueden ser relativos. Por lo anterior podemos sostener que, "novedad, valor, funcionalidad pueden ser indicadores de producto creativo, si y sólo si la creatividad valorada se orienta o considera desde la perspectiva de su efecto tangible" (p.28), ya que hay manifestaciones creativas que no llevan una funcionalidad o valor agregados, ya que pueden ser el resultado de nuevas combinaciones o disposiciones, con viejos elementos, ya sea materiales o conceptuales.

Esta última propuesta, es la que se considera más cercana al campo de la educación en artes visuales; más no deja de ser importante la referencia a un campo distinto, en el caso de Larrea, desde las ciencias económicas; para confirmar que los procesos creativos son relevantes a otros ámbitos, distintos al académico, lo que sugiere la posibilidad de tomar instrumentos y referencias que nutran desde su modelo, al del proceso que interesa en este caso. En la perspectiva de Larrea, aunque el proceso creativo se presenta como una secuencia lineal, también es cierto que tal decisión es respaldada por la estructura que promueve: momentos específicos para evaluar y analizar la factibilidad de una propuesta creativa, con la oportunidad de detectar fallas o debilidades a tiempo, lo que permite evitar seguir adelante, arrastrando perdidas económicas, gasto de tiempo y de recursos humanos y materiales. 
En la publicación "Lecciones de clase" (2018), Schwember afirma, basado en una serie de producciones artísticas realizadas en diferentes escenarios escolares de Chile, afirma que "el proceso creativo se traduce en un proceso de pensamiento complejo donde se integra la racionalidad junto con aproximaciones que acogen la intuición y los sentidos como vehículos propicios para la producción de conocimiento" (p. 70).

Para redondear el constructo sobre creatividad, producto creativo y proceso creativo, es necesario tomarlos como términos independientes, pero íntimamente enlazados por el hilo conductor llamado energía (González-Cubillán, 2008). La creatividad es la acción del proceso creativo y se representa en el producto creativo. El producto creativo es la parte visible, externa de la acción de crear a través del proceso creativo. El producto será catalogado como creativo e innovador, al momento de ser finalizado y consensuado.

Solo se hará la definición de uno de los tres elementos, producto de la decodificación, por considerar que cualquier descripción los otros dos (Alumnos y Profesores), podría conducir a una redundancia teórica.

\section{Resultados preliminares}

A continuación, se presentan en primer lugar, algunas deducciones producto de la observación participante específicamente con los tres profesores involucrados durante el estudio de caso (A, B y C).

- La dinámica que resulta en la interacción entre los estudiantes está ligada directamente al profesor con el que se encuentren.

- El comportamiento y carácter del profesor inciden directamente en el estímulo y la respuesta de los estudiantes.

- La comprensión del investigador se ve ampliada al momento de involucrarse no solo en la convivencia, sino en los ejercicios propuestos por el profesor, ya que le permite tomar el lugar del alumno. 
- Tanto directivos, como profesores y estudiantes, procesan y aceptan en tiempos distintos y de forma parcial, la participación del observador.

- Todos los laboratorios y talleres observados, son impartidos por artistas de trayectoria comprobable.

- El aspecto teórico-pedagógico, se ha desarrollado a posteriori, en la mayoría de los casos, a la inserción en la planta docente.

- La evaluación de los procesos creativos aún está poco entendida y delimitada, por lo que cada profesor observado no tiene un solo método ni herramientas específicas, y el criterio tiende a reducirse solo a la percepción personal.

- La evaluación en talleres tiende a limitarse a parámetros y rúbricas recomendadas por el programa de estudios.

- El proceso creativo se observa y es relevante para la retroalimentación entre profesores y alumnos, pero no incide directamente en la evaluación sumaria, ya que se encuentra resistencia a evaluar aspectos creativos, por considerar que eso limita la capacidad expresiva del estudiante.

- De acuerdo al grado o nivel del curso, los profesores perciben mayor libertad para involucrar aspectos conceptuales relacionados a la creatividad, es decir, en niveles iniciales se promueve un acompañamiento cercano para la obtención de habilidades técnicas relacionadas a manejo de materiales, con el avance durante la carrera de Artes y Diseño, se va incrementando una tendencia a valorar la formación de un discurso y línea de producción artística.

- Los procesos ligados a la producción, si son considerados para la evaluación de los aprendizajes, dado que involucran aplicación de técnicas y desarrollo de habilidades que son susceptibles de mediciones cuantitativas, con base en cualidades materiales.

Con base en las observaciones, se contempla la existencia de una tendencia a evitar la evaluación de los procesos creativos en el sentido directo a otorgar juicios de valor respecto de los 
trabajos/obras artísticas de los estudiantes, ya que en su mayoría coinciden en que toda evaluación crítica a los mismos, se convierte en una legitimación arbitraria y excluye a aquellos estudiantes que proponen discursos distintos a los aceptados normalmente por el gremio artístico. Algunas conclusiones parciales son:

- La evaluación formativa se revela como idónea para atender el proceso creativo. Lo anterior dado que contiene los elementos transversales y longitudinales necesarios para dar tanto estructura como flexibilidad.

- La mayoría de los profesores participantes llevan a cabo evaluaciones con diferentes grados de carácter formativo.

- Una tendencia es la de evitar dar una rúbrica con los elementos presentes en el proceso creativo, dado que se considera, le resta libertad.

- Se contempla como principal motivo para eludir cualquier tipo de rubrica, la inexistencia de un consenso profundo y claro, respecto de los elementos que debería contener un instrumento para dar seguimiento a los procesos creativos en artes visuales.

La evaluación del proceso creativo, ligado a la investigación desde las artes, aún no posee herramientas de medición bien definidas y consensuadas para obtener información instrumental, que refleje de manera general, los atributos, características y competencias logradas dentro de dicho proceso, así como la correspondiente evaluación al estudiante.

Como resultado del análisis y discusión previos, se tienen las bases para proponer una lista de acciones que se pueden tomar como modelo para el ejercicio de la evaluación a procesos creativos en programas de Artes Visuales, específicamente en los talleres prácticos que forman parte de su estructura curricular, con lo cual se da respuesta, al menos en la parte operativa, a la pregunta de investigación sobre como evaluar el proceso creativo.

El listado está ordenado en una línea de seguimiento sucesivo temporal, pero, como en toda evaluación formativa, sus elementos pueden cambiar de orden, traslaparse, repetirse o eliminarse; de acuerdo a las necesidades de cada programa y al criterio del profesor a cargo, siguiendo un poco 
la noción de "diario de viaje" al estilo de Julio Cortázar y Carol Dunlop (Dunlop y Cortázar, 1984), quienes, siguiendo una ruta de autopista, lograron encontrar que se puede, si se observa bien, descubrir nuevas cosas en cada tramo del viaje. Apoyados de las notas de viaje, sin discriminar detalles por insignificantes que parezcan a primera vista. Dicho lo anterior, a continuación las recomendaciones de actividades para estructuración de Evaluación Formativa en contextos de talleres de Artes Visuales del Nivel Superior en México.

- Revisión de antecedentes y evaluación diagnóstica para conocer el grado de habilidades, intelectuales, cognitivas y motoras, de los alumnos.

- Diálogos de manera individual con cada alumno para tener un marco referencial sobre los intereses, expectativas y habilidades específicas que el estudiante manifiesta.

- Promover una sesión al inicio del ciclo escolar para delimitar:

- El tema y actividades a desarrollar

- Relevancia de las habilidades y conocimientos que se buscan (teóricas, formales, simbólicas y lúdicas)

- Propuesta y apertura de canales de comunicación para dar seguimiento al proceso creativo de manera personal, independientemente del estadio dentro del proceso creativo

- Propuesta y presentación del esquema de la rúbrica a utilizar como instrumento de apoyo a la evaluación, el formato deberá ser resultado de la discusión y sugerencias propuestas por alumnos y profesor.

- Observar y registrar elementos inherentes a los procesos de investigación, y sean éstos evidentes o implícitos dentro del proyecto escolar que cada alumno realiza; con lo anterior el profesor contará con pruebas tangibles para fines administrativos, pero con evidencias de los procesos creativos, tanto formales como subjetivos.

- Diseñar un instrumento para registrar los errores o deficiencias que se observen al recuperar, analizar y categorizar la información obtenida de la rúbrica utilizada. 
- Con base en la información registrada, determinar si es necesario redirigir o modificar las estrategias de enseñanza; lo que le transforma además en aprendizaje, tanto para el profesor como para el alumno.

El listado anterior es presentado a continuación en un diagrama para facilitar su entendimiento, donde se muestra además las características propias del proceso, en un ciclo constante que se nutre a sí mismo; el modelo se basa en el descrito anteriormente en el capítulo del proceso creativo.

Figura 1: Modelo para evaluación del proceso creativo. Elaboración propia

\section{DIAGRÁMA PARA EVALUACIÓN DEL PROCESO CREATIVO}

- Revisión de antecedentes y evaluación diagnóstica para conocer el grado de habilidades, intelectuales, cognitivas y motoras, de los alumnos.

- Dialogos de manera individual con cada alumno para tener un marco del contexto familiar, económico, social, y cultural que le precede.
Promover una sesión al inicio del ciclo escolar para delimitar:

- El tema y actividades a desarrollar - Relevancia de las habilidades y conocimientos que se buscan (teóricas, formales, simbólicas y lúdicas)

-Propuesta y apertura de canales de

INICIAL

para dar seguimiento al proceso creativo de manera personal,

independientemente del estadio dentro de

PREVIA

Recomendaciones de actividades para estructuración de Evaluación Formativa en contextos de talleres de Diseñar un instrumento para registrar los errores o deficiencias que se observen al recuperar la información dada por la rubrica utilizada.

- Con base en la información registrada, determinar si es necesario redirigir o modificar las estrategias de enseñanza; lo que le transforma además en aprendizaje, tanto para el profesor como para el alumno. 
El modelo descrito es una propuesta flexible, ya que puede adaptarse de acuerdo a los objetivos del programa específico de cada área de estudio, los pasos que describe, son los que se revelaron como ideales para el proceso de evaluación al proceso creativo en talleres de artes visuales, sin embargo se pueden adecuar a otras áreas (arquitectura, escénicas, diseño y todas aquellas donde exista un proceso creativo), manteniendo la estructura metodológica central, se pueden embonar otros objetivos específicos, redactados de manera que puedan beneficiarse del modelo propuesto.

Dentro de su carácter flexible, el modelo también es alternativa para adecuarse a otras áreas y contextos (arquitectura, escénicas, diseño y todas aquellas donde exista un proceso creativo), manteniendo la estructura metodológica central, se pueden embonar otros objetivos específicos, siempre que se respete el esquema general para conservar la estructura y así beneficiarse del modelo propuesto

\section{Conclusiones y recomendaciones}

El cierre para el artículo presente, gira en torno a las particularidades reveladas a través de contrastes entre lo observado y la postura de los teóricos que fundamentan la investigación realizada. El camino será partir desde las particularidades de los casos de estudio. La primera, y quizás la que con mayor contundencia se reveló una y otra vez, es la arraigada idea de que evaluar es equivalente a calificar y, por tanto, si se busca emitir un juicio por medio de una nota, resultado de un examen o ejercicio final; tal evento se puede transformar en palabras de Mazur: un "asesino para la creatividad" (Mazur, 2020).

La siguiente pregunta que surge es, ¿por qué es relevante la creatividad y sus productos dentro del contexto de estudio? Una de las respuestas es que el valor de la creatividad está implícito no solo en las artes, sino en prácticamente todos los ámbitos de la vida; se requiere de creatividad para idear nuevas formas de pensamiento (pensamiento creativo); el proceso creativo está presente al momento de planear estrategias de comerciales; investigación para nuevos 
medicamentos; producción de vehículos automotores más eficientes y estéticos; y la lista puede continuar desde cada ámbito en el mundo.

En el caso de instituciones académicas, la evaluación forma parte de los procesos de enseñanza y de aprendizaje, donde, por lo general (al menos en los casos observados), solo se usa como validación de los aprendizajes conseguidos, dejando de lado sus posibles aportes también para el proceso de enseñanza.

Para lograr integrar ambos procesos y obtener beneficios de la evaluación, la evaluación formativa se revela como la herramienta idónea, ya que desde ella pueden integrarse ambos elementos, de manera que no solo para ayudar al alumno a conseguir los objetivos planteados en el curso, sino para aprender de los errores, con lo que se logra nutrir al que aprende y al que enseña; el que aprende (el alumno), se hace consciente, con ayuda del profesor, de las fallas y dificultades con que se enfrenta; y el que enseña (el profesor), aprende de tal intercambio, auxiliado en su "diario de viaje", desde donde puede plantear nuevas estrategias didácticas.

El listado propuesto para ayudar a la evaluación del proceso creativo, se revela como una herramienta incompleta, con la intención de abrir la discusión a posibles modificaciones, anexos, correcciones y adecuaciones, de acuerdo a los intereses y necesidades de investigadores futuros; pero una aseveración derivada de la Tesis en conclusión, es que los procesos creativos son susceptibles de ser evaluados; y en el contexto educativo, deben serlo, si se pretende dar pasos para la completa validación e integración del estudio de las artes en los programas educativos, con lo que puedan transitar de ser considerados solo un área de expresión artística y esparcimiento emocional, a un elemento con múltiples aplicaciones prácticas y funcionales, con importancia equivalente a estudios de ingeniería, economía, medicina, entre otros, tradicionalmente considerados como carreras de aplicaciones prácticas para la vida.

Para dar cierre, se deja el pensamiento de Luis Camnitzer (2012), "Se puede afirmar que la enseñanza del arte se dedica fundamentalmente a la enseñanza sobre cómo hacer productos y como funcionar como artista, en lugar de cómo revelar cosas. " El concepto medular de la frase nos lleva a reflexionar sobre la validez de aprender y enseñar desde el rigor de la ciencia, en éste caso 
nacida en las instituciones educativas, y una vez dominados los elementos fundamentales, incentivar saltos al vacío, utilizando el "sombrero" de la creatividad (De Bono, 1988), hacia terrenos inexplorados, que es donde aguardan posibilidades infinitas y que permitan que nuevos conocimientos, habilidades maneras de ser en el mundo, sean revelados.

\section{Referencias}

Bauman, Z. (2005). Modernidad liquida. Buenos Aires, Buenos Aires. Argentina: Fondo de Cultura Económica de España.

Camnitzer, L. (21 de marzo de 2012). La enseñanza del arte como fraude. [comunicación en página web] http://blogs.fad.unam.mx/asignatura/ingrid sosa/wp-content/uploads/2017/01/10.La-Ensen\%CC\%83anza-del-arte-como-fraude Camnitzer.pdf

De Bono, E. (1988). Seis sombreros para pensar. Buenos Aires, Argentina.

De Vincenzi, A. (abril de 2009). La práctica educativa en el marco del aula taller. Educación y desarrollo (10), 41-46.

Dunlop, C., y Cortázar, J. (1984). Los autonautas de la cosmopista. http://biblioteca.d2g.com

González-Palacios, T. E. (2014). La creatividad: proceso, elementos y valoración de efecto en carreras de la Universidad Eloy Alfaro . ECA Sinergia. Portoviejo, Manabí, Ecuador.

Herrán-Gascón, A. d. (abril de 2014). Creatividad y formación radical en inclusiva: cuando la creatividad no sirve para nada. En: P. J. García-Sempere, \& P. Tejeda-Romero, Edits) Granada, Granada, España: Universidad de Granada.

Jiménez-Fontana, R., García-González, E., Azcárate, P., Navarrete, A., \& Cardeñoso, J. M. (2016). La Teoría Fuandamentada como estrategia de análisis de los datos: caracterización del proceso. 5a Congreso Iberoamericano en Investigación Cualitativa. L. 356-365. Porto: Atas.

Mazur, E. (16 de marzo de 2020). Observatorio de innovación educativa. (T. d. Monterrey, Productor) Observatorio de innovación educativa: https://observatorio.tec.mx/edunews/entrevista-eric-mazur-evaluacion-aprendizaje 
Michaud, Y. (2007). El arte en estado gaseoso. Ensayo dobre el triunfo de la estética. México, D.F., México: Fondo de Cultura Económica.

Mjelde, L. (2015). Las propiedades mágicas de la formación en el taller. Montevideo, Uruguay.

Romero, M. (2011). Orientaciones para la elaboración de programaciones en artes. En A. Giráldez, L. Pimentel y Coords., Educación artística, cultura y ciudadanía. 135-146. Madrid, Madrid, España: Organización de Estados Iberoamericanos para la Educación, la Ciencia y la Cultura $(\mathrm{OEI})$.

Schwember Bustamante, F., Wolff Rojas, A., \& Cecilia Palma Galería de Arte. (2007). Francisco Schwember : Obra pictórica (Catálogo de exposición de arte). Santiago, Chile: Galería de Arte Cecilia Palma

Spentsas, A. C. (2017). Siendo espectador (II). La experiencia del espectador como forma de participación no reglada. DOSSIER (25), 204-220

Storr, R. (2009). Dear Colleague. En S. Madoff y Madoff (Ed.), Art school (propositions for the 21st century) 59-60. Cambridge, Massachusetts, USA: MIT Press.

Strauss, A. y Corbin, J. (diciembre de 2002). Bases de la investigación cualitativa. Técnicas y procedimientos para desarrollar la teoríafundamentada. Medellín, Antioquia, Colombia. 